\title{
Regulación de la respuesta inmune durante la infección por Mycobacterium tuberculosis Artícullo de revisión
}

\author{
Sánchez-Villa José Diego*, Preciado de Santos Miguel *
}

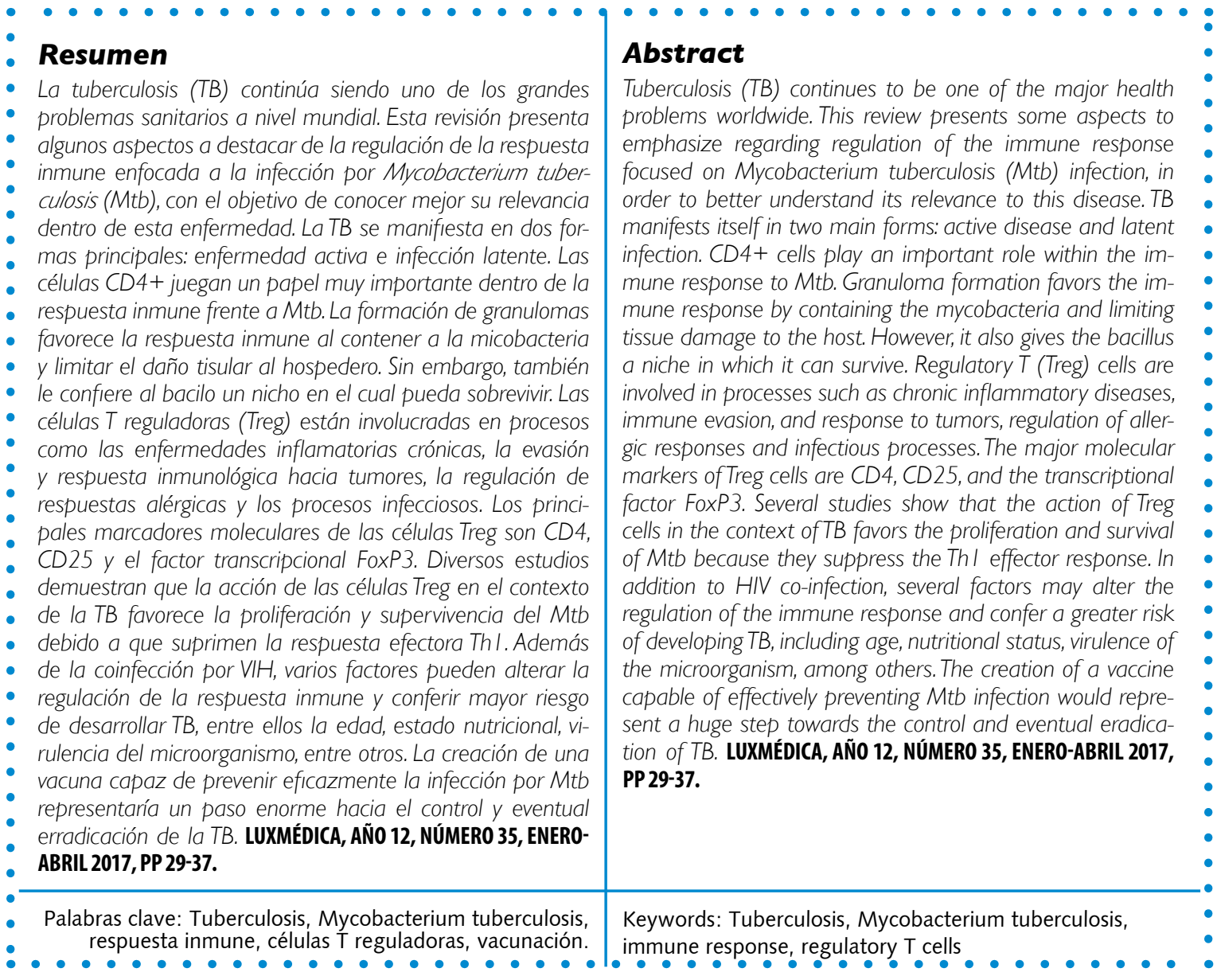

Médico Cirujano, egresado de la carrera de Medicina del Centro de Ciencias de la Salud de la Universidad Autónoma de Aguascalientes.

Fecha de recibido: 25 de septiembre 2016

Fecha de aceptación: 15 de diciembre 2016

Correspondencia: Dr Alejandro Rosas Cabral, Departamento de Medicina, edificio 107 planta alta, Centro de Ciencias de la Salud, Universidad Autónoma de Aguascalientes. Avenida Universidad \#940, Ciudad Universitaria Código Postal 20131, Aguascalientes, Ags., México. Teléfono 01449 9108436 correo electrónico drrosascabral@gmail.com 


\section{Introducción}

Uno de los grandes problemas sanitarios tanto en México como en muchas otras regiones del mundo, especialmente aquellas en vías de desarrollo, sigue siendo la tuberculosis (TB). La TB es una enfermedad infecciosa, crónica, causada principalmente por el bacilo Mycobacterium tuberculosis. Típicamente afecta a los pulmones pero también puede afectar otros sitios. Se contagia por vía aérea cuando un paciente infectado expele los bacilos, por ejemplo con la tos. En promedio, una pequeña proporción (5-15\%) de los 2 a 3 billones de personas en el mundo infectadas con el bacilo tuberculoso desarrollarán la enfermedad durante su vida. La probabilidad de padecer la enfermedad se incrementa si el paciente esta coinfectado con el virus de la inmunodeficiencia humana (VIH). Según el reporte más reciente de la Organización Mundial de la Salud (OMS), para el año 2014, se estimó una incidencia global de 9.6 millones de nuevos casos y de 1.5 millones de muertes ocasionadas por $\mathrm{TB}$, con lo que se mantiene como una de las causas principales de muerte a nivel mundial. ${ }^{1}$

La regulación de la respuesta del sistema inmune, dada principalmente por las células $T$ reguladoras (Treg), juega un papel crucial en la enfermedad. Sin embargo, se ha visto que esta regulación favorece la replicación y supervivencia del Mtb. Se han realizado múltiples estudios buscando maneras de controlar esta respuesta reguladora, que puedan conducir a nuevos enfoques terapéuticos o adecuaciones en el esquema de vacunación con mayor efectividad. ${ }^{2}$

Esta revisión presenta algunos aspectos a destacar de la regulación de la respuesta inmune enfocada a la infección por $M$. tuberculosis, con el objetivo de conocer mejor su relevancia dentro de esta enfermedad.

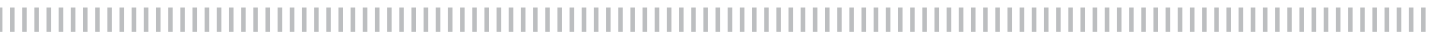

\section{Generalidades de la respuesta inmune frente a M. tuberculosis}

La TB se manifiesta en dos formas principales: enfermedad activa e infección latente. Esta última no es transmisible ni presenta sintomatología clínica, debido a que la respuesta inmune es capaz de contener el crecimiento del patógeno pero no de eliminarlo, de manera que la bacteria persiste en el organismo manteniendo una baja o nula actividad proliferativa. La importancia de la TB latente radica en el riesgo de reactivación de la infección, incluso después de años, favorecida por condiciones que supriman al sistema inmunológico. ${ }^{3}$
Las células CD4+ juegan un papel importante dentro de la respuesta inmune frente a Mtb. En primera instancia, otorgan protección frente al patógeno, pero también contribuyen al establecimiento de una infección crónica, las reactivaciones de la enfermedad y provocan mayor daño tisular. ${ }^{4}$ Asimismo, su depleción, como en el síndrome de inmunodeficiencia adquirida (SIDA), conduce a una mayor susceptibilidad a la infección. Las células CD8+ también tienen una función importante, ya que son capaces de eliminar células infectadas por Mtb gracias a la producción de moléculas citotóxicas, como las perfo- 
rinas y las granzimas, además de la producción de citocinas capaces de modular el microambiente. ${ }^{5}$ Por su parte, los posibles mecanismos a través de los cuales la inmunidad humoral pueden contribuir a la defensa frente a TB incluyen la opsonización, que promueve la fagocitosis, el aclaramiento de antígenos inmunomoduladores liberados por Mtb, entre otros. ${ }^{6}$

Los modelos experimentales de infección por $M$ tb realizados en ratones para reproducir los diferentes patrones de respuesta inmune desencadenados, como el propuesto por Hernández-Pando y cols (1996), demuestran que el progreso de la infección por la micobacteria en el pulmón comprende dos fases bien diferenciadas: la primera durante los primeros 28 días de la infección, dominada por un patrón de respuesta inmune de tipo Th1, en presencia de citocinas como interferón-y (IFN-y) e interleucina-12 (IL-12), que se refleja en el tejido pulmonar con infiltrado inflamatorio pericapilar, peribronquial e intersticial, con la formación y subsecuente desarrollo de granulomas; y la segunda fase a partir de los 28 días de la infección, en la cual existe un equilibrio entre la respuesta inmune de tipo Th1 y Th2, pues aumenta la producción de citocinas como IL-4 entre otras, con el desarrollo de ocupación alveolar por infiltrado inflamatorio y detritus celulares (áreas neumónicas), necrosis focal, fibrosis y la progresión de la enfermedad. La respuesta Th1 participa principalmente en la activación de macrófagos y en la hipersensibilidad retardada, mientras que las células Th2 inducen la diferenciación de las células B y parecen antagonizar la respuesta Th1. ${ }^{4}$

La estructura más importante de esta respuesta inmune evocada por el Mtb es el granuloma, que es un conglomerado organizado de células inmunes que contienen bacilos viables. El granuloma favorece la respuesta inmune al contener a la micobacteria y limitar la extensión de la inflamación y el daño tisular al hospedero. Sin embargo, también le confiere al bacilo tuberculoso un nicho en el cual puede prolongar su supervivencia., ${ }^{2,7}$ (figura 1)

Figura 1 Estructura y componentes celulares del granuloma tuberculoso.

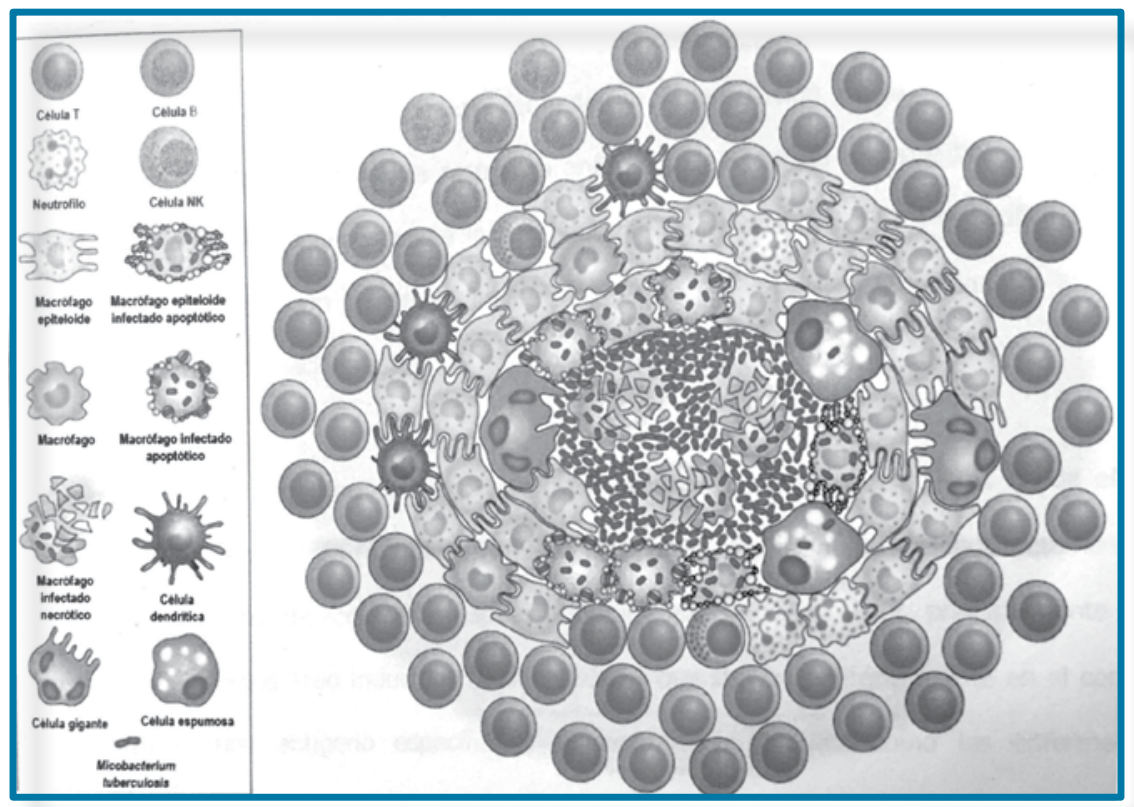

Imagen tomada de Ramakrishnan L. Revisiting the role of the granuloma in tuberculosis. Nature, Inmunology 2012;12:352-366. 
Las micobacterias en estado de latencia pueden residir no sólo en macrófagos dentro de granulomas pulmonares antiguos, sino también en células de diferentes órganos. Barrios y cols (2012) demostraron esto al encontrar, en tejidos obtenidos por autopsia de individuos que fallecieron por causas distintas a TB, ADN micobacteriano localizado tanto en pulmones como en sitios extrapulmonares, entre las que destacan bazo, riñón e hígado; por lo que es de suponer que el control inmunológico efectivo de la TB incluye además la fagocitosis por macrófagos y la formación de granulomas. $^{8}$

\section{Células $T$ reguladoras}

Gracias a las nuevas tecnologías, la caracterización de los linfocitos T CD4+ se ha expandido al reconocimiento de más subtipos además de los Th1 y Th2, que incluyen los Th17, las células Treg, entre otros. ${ }^{9}$ Las células Treg están involucradas predominantemente en la regulación de las funciones inmunes fundamentales, como la autotolerancia y la respuesta antitumoral. Evidencia reciente ha encontrado su participación en la regulación de la respuesta inmune durante las infecciones. El papel específico de estas células Treg durante una infección puede ser limitar respuestas Th1 agresivas inducidas por antígenos microbianos, previniendo inflamación excesiva y daño tisular. ${ }^{10}$

Existen diferentes subtipos de linfocitos Treg. Una clasificación adecuada es la que los divide en dos grupos: los linfocitos Treg naturales (o nativos), que se originan y adquieren su función directamente en el timo y tienen la propiedad de regular la respuesta inmune vía contacto celular, principalmente hacia autoantígenos; y los linfocitos Treg inducidos (o adaptativos) que surgen periféricamente en el contexto de una respuesta inmune antígeno específica y relacionados a eventos como las enfermedades inflamatorias crónicas, la evasión y respuesta inmunológica hacia tumores, la regulación de respuestas alérgicas y los procesos infecciosos. ${ }^{10-12}$ (Figura 2)

Figura 2: Esquema de la producción de células $\mathbf{T}$ reguladoras.

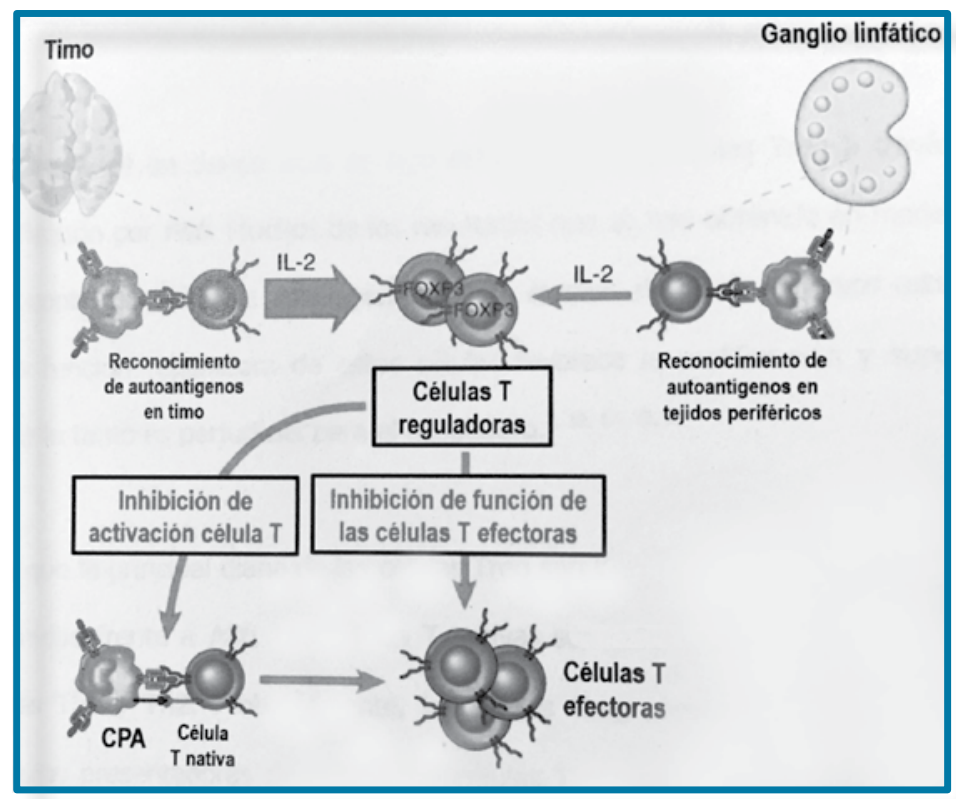

(IL-2: Interleucina-2; CPA: Célula presentadora de antígeno). Imagen tomada de Abbas y colaboradores. Cellular and molecular immunology. 6 a Ed. Elsevier www.studentconsult.com 
Los principales marcadores moleculares de las células Treg han sido CD4+, alta expresión de CD25+ (cadena alfa del receptor de Interleucina-2), FoxP3+ (el factor transcripcional característico de estas células, el cual es un marcador intracelular) ${ }^{13}$ y CD127- (receptor de interleucina-7). Recientemente se ha mencionado al CD39 como otro marcador de las células Treg, incluso más específico que los demás marcadores. ${ }^{14}$ También se habla del CD52 como marcador y molécula efectora de otro subtipo de linfocitos Treg, los cuales no tienen un perfil de expresión molecular clásico. ${ }^{15}$

Las células Treg actúan de manera diversa contra un agente infeccioso, dependiendo del patógeno que se trate. Se ha visto que en infecciones por virus, como el $\mathrm{VIH}$, virus herpes simple, virus de la hepatitis $C$, entre otros, la depleción de estas células provoca una infección más grave que la comparada con sujetos inmunocompetentes. En cambio, con algunas infecciones bacterianas en su fase crónica, como Helycobacter pylori o Mtb, su depleción provoca una respuesta inmune más eficaz contra los patógenos, ${ }^{2}$ debido a que las Treg suprimen la respuesta Th1 en contra del agente causal. ${ }^{10}$

Una de las enfermedades en donde más se han estudiado a las células Treg a través de diferentes métodos, es la infección por Mtb. Muchos de los resultados que se han obtenido en modelos animales de TB no han sido concluyentes, pero existen datos en diversos estudios en donde probablemente la función reguladora de estas células favorece la proliferación y supervivencia de la micobacteria y, por lo tanto, es perjudicial para el hospedero. 2, 16-19

Se conoce que la principal diana o células blanco de las Treg son las células $T$ CD4+ y CD8+, las cuales son clave en la inmunidad frente a Mtb. Las células T nativas son más sensibles a las células Treg que los linfocitos efectores Th1 y Th2. Probablemente, las células Treg previenen la formación de la sinapsis inmune entre células presentadoras de antígeno y células $T$ efectoras. Se cree que este mecanismo es debido a la interacción competitiva del activador negativo CTLA-4 (antígeno 1 del linfocito T citotóxico, por sus siglas en inglés) con moléculas coestimuladoras como B7 en la superficie de las células diana, provocando así anergia celular. Cuando una célula Treg entra en contacto con su célula diana, esta última adquiere funciones supresoras y comienza a secretar citocinas inhibidoras que reducen la actividad proliferativa y secretora de otras células diana secundarias, con lo que el mantenimiento de una respuesta inmune efectiva será poco probable y llevará a un curso desfavorable de la enfermedad. ${ }^{16}$

Ribeiro-Rodríguez y cols (2006) encontraron que la depleción de las células Treg mejora la producción de células T secretoras de INF-y en pacientes recién diagnosticados de $T B$, indicando que la cantidad incrementada de células T CD4+CD25+ no es sólo un reflejo de activación inmune excesiva durante la $T B$, sino que también tienen un papel en el control de la respuesta inmune. ${ }^{17}$

En un modelo experimental, ScottBrowne y cols (2007) compararon por tinción inmunohistoquímica la acumulación de células que expresan FoxP3 entre ratones infectados por Mtb contra ratones no infectados. Demostraron que los ratones no infectados tenían muy pocas células Treg (que expresan FoxP3), observadas en regiones peribronquiales y perivasculares, y casi ninguna en otras áreas del parénquima pulmonar, a diferencia de los ratones infectados, que presentaban una gran cantidad de células Treg distribuidas tanto a nivel peribronquial y perivascular como en granulomas (figura 3). Asimismo, observaron agregados de estas células en ganglios linfáticos, concluyendo que su acumulación en todas estas zonas corre paralela a la acumulación de las células T efectoras. ${ }^{2}$ 
Figura 3. Localización de células Treg en pulmones de ratones infectados.

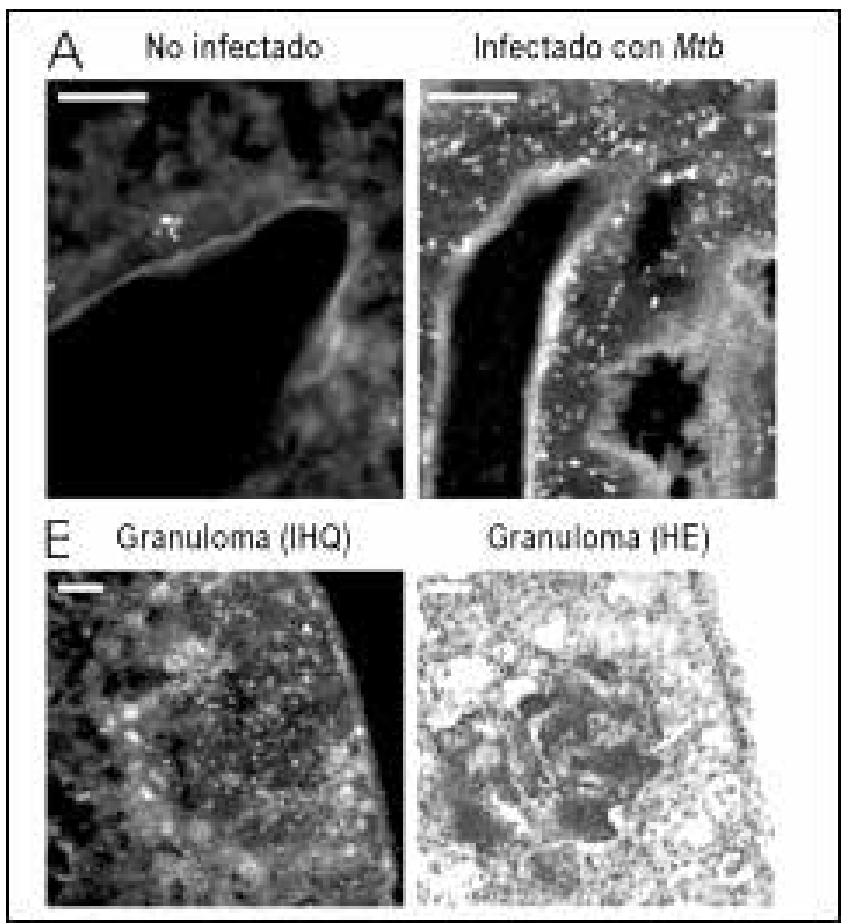

(A) Cortes representativos mostrando áreas perivasculares de pulmones de ratones no infectados o infectados con Mtb (115 días tras la infección) marcados con anti-FoxP3 (verde); (B) Cortes mostrando granulomas parenquimatosos de un ratón infectado con Mtb (60 días tras la infección) marcados con anti-FoxP3 (verde) y CD4 (rojo; izquierda) y hematoxilinaleosina (derecha).

(IHC: tinción por inmunohistoquímica; HE: tinción por hematoxilinaleosina).

Figura tomada de Scott-BrowneJ, Shafiani S, Tucker-Heard g y cols. Expansion and function of foxp3-

expressing T regulatory cells during tuberculosis. The Journal of Experimental medicine 2007;204:2159-2169

Por otra parte, cuando la presencia de las células Treg es cuantificada con base en su expresión del marcador CD39+, se encuentra un mayor número circulante de estas células en individuos con TB activa que en aquellos con TB latente, indicando una posible correlación entre la actividad de dichas células y la fase en la cual se encuentre la enfermedad. ${ }^{19}$

Es muy importante saber si las células Treg que responden frente a la infección por Mtb son patógeno-específicas. Si la mayoría no lo son, y simplemente responden a la inflamación y daño tisular, entonces el manipular su respuesta tendrá poco impacto en prevenir o tratar la infección y pudiera conferir mayor susceptibilidad a enfermedades autoinmunes. Por otro lado, si las células Treg que se generen durante la infección son específicas frente a antígenos de $M t b$, es posible lograr beneficios clínicos al detener su respuesta, especialmente durante la inmunización. ${ }^{2}$

\section{Otros aspectos}

Además de la coinfección por $\mathrm{VIH}$, varios son los factores que contribuyen a alteración en la regulación de la respuesta inmune y a un mayor riesgo de desarrollar $T B$, entre los que se encuentran la edad, el estado nutricional, la virulencia del microorganismo, uso de fármacos, etc. A 
continuación, mencionaremos aspectos a destacar de algunos de ellos.

\section{Tuberculosis en los niños y vacunación}

Los niños pertenecen a un grupo relativamente susceptible a desarrollar enfermedad progresiva y diseminación extrapulmonar posterior a la infección por Mtb. Los mecanismos implicados en esta susceptibilidad se están estudiando arduamente en la actualidad. Por ejemplo, el grupo de Pavan-Kumar y cols (2011) llevaron a cabo un estudio en el cual analizaron los perfiles de expresión de citocinas en niños diagnosticados con $T B$, encontrando que tanto las respuestas de tipo Th1, como las Th2 y Th17 se encontraban disminuidas, ya sea posterior o no a la estimulación in vitro con antígenos de Mtb, en comparación a niños sanos, siendo esta disminución más marcada en niños con TB neurológica, sugiriendo un papel crucial de estos tres perfiles de citocinas en la protección contra TB pediátrica, en especial en aquellos con diseminación extrapulmonar. ${ }^{9}$ Esto difiere con algunos modelos experimentales en animales que sugieren que un incremento en IL-4 y otras citocinas con perfil Th2 serían perjudiciales en la resistencia del hospedero a TB.

Una vacuna capaz de prevenir eficazmente la infección por Mtb representaría un paso enorme hacia el control y eventual erradicación de la TB. Hoy en día, la única vacuna autorizada contra TB es la Mycobacterium bovis BCG (Bacilo de CalmetteGuerin), la cual provee una pobre protección frente a formas pulmonares de TB, mientras que para formas extrapulmonares graves, como la miliar o meníngea, provee una mejor protección. ${ }^{6}$

Jaron y cols (2008) observaron que la atenuación de las células Treg junto con la aplicación de la vacuna BCG, provocó un impacto positivo, pero limitado, en la capacidad protectora de la vacuna frente a Mtb. Por lo tanto, se debe tener en cuenta que, aunque la acción de las células T reguladoras no representan la mayor causa de eficiencia limitada de la BCG, el impacto de esta población celular es significativo y medible. ${ }^{20}$

Se ha propuesto que las células Th17 participan en la inducción de memoria específica contra Mtb y la respuesta protectora posterior a la vacunación. Sin embargo, Pavan-Kumar y cols (2011), en su estudio analizaron también la inducción de respuestas Th1, Th2 y Th17 en el contexto de niños enfermos de TB vacunados con $B C G$, en el cual, no se encontraron diferencias significativas en ninguna de las citocinas, lo que indica que la BCG tiene muy poca, si es que ninguna, capacidad de inducir una respuesta inmune diferencial en tuberculosis pediátrica. ${ }^{9}$

Algunos autores han propuesto que la aparente falta de eficacia de la vacuna BCG reside en una estrategia de administración inadecuada, y que la administración por aerosol podría ser la única forma de activar la población de células T pulmonares capaces de inhibir el crecimiento temprano de la micobacteria y que no se alcanza por la vía parenteral clásica. Basándose en modelos experimentales previos, una alternativa óptima requiere de la administración tanto parenteral como inhalada de la vacuna. ${ }^{6}$

En Estados Unidos, en abril de 2014, se llevó a cabo la primera reunión que se concentró específicamente en la posibilidad de usar vacunas en aerosol contra la TB, explorando el potencial de esta vía en prevenir la infección por el Mtb, en prevenir el desarrollo de TB activa en aquellos con infección latente, o como inmunoterapia para individuos con TB activa. El beneficio de esta estrategia está apenas comenzando a explorarse. ${ }^{6}$

\section{Tuberculosis en relación a diabetes mellitus y otros factores hormonales}

Dada la creciente prevalencia de la diabetes mellitus tipo 2 (DM2) en muchas regiones 
del mundo, es probable que esta enfermedad crónica contribuya al aumento de casos de TB, al aumentar la susceptibilidad del hospedero, como lo han propuesto recientemente Delgado y cols (2015). En México, de 2000 a 2012, la tasa de incidencia de TB pulmonar asociada a DM se incrementó en $82.64 \%$, en contraste con la tasa de incidencia de TB en individuos sin DM, la cual disminuyó en $26.77 \%$. Aquellos con diagnóstico previo de DM tuvieron una mayor probabilidad de falla al tratamiento antituberculoso comparada con quienes no tienen DM. Aunque la fisiopatología de la susceptibilidad a TB en pacientes con DM no ha sido del todo aclarada, se han descrito mecanismos que involucran tanto a la regulación de la inmunidad innata como a la adaptativa. ${ }^{21,22}$ Estos datos indican que las estrategias de control y prevención de DM deben ser integradas a los programas para control de TB. ${ }^{21}$

La relación entre hombres y mujeres que padecen TB pulmonar es de $7 / 3 \mathrm{a}$ favor de los hombres. Estudios epidemiológicos en países en desarrollo muestran que los hombres sufren enfermedad más severa, mayores tasas de reactivación de infección latente y peores resultados al tratamiento. ${ }^{23}$ Es de llamar la atención que esta diferencia entre géneros se ve en adultos de todas las edades, pero no en niños o adolescentes jóvenes. Esto sugiere, además de los factores socioculturales, la participación de factores biológicos, en particular los bien conocidos efectos reguladores que las hormonas sexuales esteroideas tienen sobre las células del sistema inmune, considerando que la testosterona debilita la activación de macrófagos y la producción de citocinas proinflamatorias, mientras que los estrógenos son mediadores en la inducción proinflamatoria. ${ }^{23}$

Bini y cols (2014) llevaron a cabo uno de los primeros modelos experimentales en los cuales se demostró esta relación entre hormonas sexuales y TB, al observar en su estudio que los ratones macho son más susceptibles a TB que las hembras, y que esto se prevenía por medio de la castración quirúrgica de los machos, proponiendo que los niveles de testosterona pueden ser un factor independiente de susceptibilidad a TB. ${ }^{23}$

\section{Tuberculosis multidrogo resistente}

Otro de los problemas que en años recientes ha cobrado mucha relevancia en el manejo y control de la TB es la aparición de cepas multidrogo resistentes (MDR), definida como la resistencia conjunta a isoniazida y rifampicina. El tratamiento de la TB en estos casos es más largo y requiere fármacos más tóxicos y más caros. En cuanto a la participación de la regulación de la respuesta inmune, investigaciones recientes sugieren que las cepas de micobacterias MDR, tienen propiedades especiales con respecto a la inducción de células Treg CD4+CD25+ con actividad supresora. ${ }^{16}$

\section{Conclusiones}

La regulación de la respuesta inmune frente a Mtb es un proceso complejo y variado. La presencia y participación de las células Treg en todas las variantes y formas clínicas de TB nos permite suponer que llevan a cabo una función inmunosupresora importante dentro de esta enfermedad. Son necesarias más investigaciones y experimentos para comprender mejor la relación de estas células y de muchos otros factores en la regulación inmunológica dentro de la TB, para así poder llevar estos conocimientos a la aplicación clínica para pacientes que padezcan esta enfermedad. 


\section{Bibliografía}

1. World Health Organization. Global Tuberculosis Report 2015, 20th edition. ISBN 9789241565059.

2. Scott-Browne J. Shafiani S. Tucker-Heard G. et.al. Expansion and function of Foxp3-expressing T regulatory cells during tuberculosis. The Journal of Experimental Medicine 2007; 204: 2159 - 2169.

3. Barrios J. Castañón M. Flores M. Hernández R. Aspectos biológicos, clínicos y epidemiológicos de la tuberculosis latente. Salud Pública Mex 2010; 52:7078.

4. Hernández-Pando R. Orozcoe H. Sampieri A. et.al. Correlation between the kinetics of Th1/Th2 cells and pathology in a murine model of experimental pulmonary tuberculosis. Immunology 1996; 89: 2633.

5. Day C. Moshi N. Abraham D. et.al. Patients with Tuberculosis Disease Have Mycobacterium tuberculosis-Specific CD8 T Cells with a Pro Apoptotic Phenotype and Impaired Proliferative Capacity, Which Is Not Restored following Treatment. PLOS ONE 2014; 9(4): 1 - 12. e94949. doi:10.1371/ journal. pone.0094949.

6. Schrager L. and Sizemore C. Conference report: Developing aerosol vaccines for Mycobacterium tuberculosis: Workshop proceedings. Conference report, Vaccine 2015; 33: 3038 - 3046.

7. Ramakrishnan L. Revisiting the role of the granuloma in tuberculosis. Nature, Immunology 2012; 12: 352 $-366$.

8. Barrios J. Saqui M. Jeyanathan M. Extrapulmonary Locations of Mycobacterium tuberculosis DNA During Latent Infection. Journal of Infectious Diseases 2012; 1 - 12. DOI: 10.1093/infdis/jis381

9. Pavan N. Anuradha R. Suresh R. et.al. Suppressed Type 1, Type 2, and Type 17 Cytokine Responses in Active Tuberculosis in Children. Clinical And Vaccine Immunology 2011; 18: 1856-1864.

10. Jaramillo F.J. Gómez L.M. Anaya J.M. Células T reguladoras, infección y autoinmunidad: implicaciones en terapéutica. Infectio 2006; 10(3): 178-185

11. Curotto de Lafaille M. Lafaille J. Natural and Adaptive Foxp3+ Regulatory T Cells: More of the same or a Division of Labor? Immunity 2009; 30: 626-635.

12. Abbas et al. Cellular and molecular immunology. $6 \mathrm{e}$. Elsevier. - www.studentconsult.com

13. Gonzalez J.L. Duque V. Velasquez M. FOXP3: Controlador maestro de la generación y función de las células reguladoras naturales. Inmunología 2010; 29: $74-84$.

14. T. Chiacchio T. Casetti R. Butera O.

15. et.al. Characterization of regulatory $T$ cells identified as CD4+CD25highCD39+ in patients with active tuberculosis. Clinical and Experimental Immunology 2009; 156: 463-470.

16. Churina E.G. Urazova O.I. Novitskiy V.V. The Role of Foxp3-Expressing Regulatory $\mathrm{T}$ Cells and $\mathrm{T}$ Helpers in Immunopathogenesis of Multidrug Resistant Pulmonary Tuberculosis. Tuberculosis Research and Treatment 2012; Article ID 931291, doi:10.1155/2012/931291.

17. Ribeiro R. Resende T. Rojas R. et.al. A role for $\mathrm{CD} 4+\mathrm{CD} 25+\mathrm{T}$ cells in regulation of the immune response during human tuberculosis. Clinical and Experimental Immunology 2006; 144: 25-34.

18. Quinn K. McHugh R. Rich F. et.al. Inactivation of $\mathrm{CD} 4+\mathrm{CD} 25+$ regulatory $T$ cells during early mycobacterial infection increases cytokine production but does not affect pathogen load. Immunology and Cell Biology 2006; 84: 467-474.

19. Kim K. Perera R. Tan D. B. A. et al. Circulating mycobacterial-reactive $C D 4+T$ cells with an immunosuppressive phenotype are higher in active tuberculosis than latent tuberculosis infection. Tuberculosis (Edinb). 2014; 94 (5): 494-501.

20. Jaron B. Maranghi E. Leclerc C. Majlessi L. Effect of Attenuation of Treg during BCG Immunization on Anti-Mycobacterial Th1 Responses and Protection against Mycobacterium tuberculosis. PLoS ONE 2008; 3: 1 - 10.

21. Delgado-Sánchez G. García-García L. CastellanosJoya M. et al. Association of Pulmonary Tuberculosis and Diabetes in Mexico: Analysis of the National Tuberculosis Registry 2000- 2012. PLoS ONE 2015; 10(6): 1 - 14. e0129312. doi:10.1371/ journal. pone. 0129312 .

22. Restrepo B. Schlesinger L. Host-pathogen interactions in tuberculosis patients with type 2 diabetes mellitus. Tuberculosis (Edinb) 2013; 93(0): S10 S14.

23. Bini E. Mata D. Marquina B. et.al. The Influence of Sex Steroid Hormones in the Immunopathology of Experimental Pulmonary Tuberculosis. PLOS ONE 2014; 9(4): 1 - 9. e93831. doi:10.1371/journal. pone.0093831. 